Medicina, Ribeirão Preto,

35: 389-391, jul./set. 2002

\title{
Grupo de Trabalho para Avaliação da Comissão de Graduação
}

\author{
José Fernando de Castro Figueiredo ${ }^{1}$; Maria de Lourdes Veronese Rodrigues ${ }^{2}$ \& \\ Luiz Ernesto de Almeida Troncon ${ }^{1}$
}

Docentes. 'Departamento de Clínica Médica. ${ }^{2}$ Departamento de Oftalmologia, Otorrinolaringologia e Cirurgia de Cabeça e Pescoço. Faculdade de Medicina de Ribeirão Preto - USP

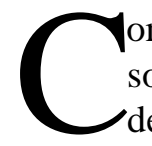

omo resultado final de um longo processo de estudos e discussões, a Faculdade de Medicina de Ribeirão Preto da Universidade de São Paulo (FMRP-USP), em reunião de sua Congregação, realizada em 15 de maio de 1992, decidiu que a Comissão de Graduação iniciasse, em 1993, a implantação de nova estrutura curricular para o Curso de Graduação em Medicina. A nova estrutura era composta de longa série de objetivos e inovações, o que incluía um processo denominado AVALIAÇÃO TERMINAL DE COMPETÊNCIAS CLÍNICAS DOS GRADUANDOS EM MEDICINA. O processo, que atendia ao objetivo geral de prover contínua avaliação da eficácia do novo currículo, por decisão da Comissão de Graduação, foi aplicado, pela primeira vez, aos graduandos de 1993.

$\mathrm{O}$ grupo encarregado de gerenciar o processo de avaliação foi constituído pelos Profs. Drs. Luis Ernesto de Almeida Troncon (Coordenador de 1993 a 1997), José Fernando de Castro Figueiredo (Coordenador de 1997 a 2001); Maria de Lourdes Veronese Rodrigues; Carlos Eli Piccinato; Luis César Peres; Ana Raquel Lucato Cianflone (pedagoga do Centro de Apoio Educacional e Psicológico - CAEP); e Maria de Fátima Aveiro Colares (Psicóloga do CAEP).

Uma das primeiras preocupações da equipe foi a relacionada com a capacitação de seus membros no processo de avaliação, sendo programados seminários e grupos de discussão, coordenados por especialistas nas áreas de Educação Médica e de Avaliação, como os Profs. Drs. Heraldo Vianna (Fundação Carlos Chagas), Lisete Diniz Ribas Casagrande (Faculdade de Filosofia Ciências e Letras de Ribeirão PretoUSP), Eduardo Marcondes e Ernesto Lima Gonçalves (Faculdade de Medicina da USP). Em duas situações, houve necessidade de assessoria de outros profissionais da área de Psicologia: avaliação da relação médico-paciente e elaboração e validação de escala de atitudes. Na primeira, contou-se com a colaboração do Prof. Dr. Marco Antonio de Castro Figueiredo, da Faculdade de Filosofia Ciências e Letras de Ribeirão Preto - USP, tanto na elaboração do instrumento quanto na avaliação de alunos. No que diz respeito à avaliação de atitudes, o grupo recebeu orientação do Prof. Dr. José Augusto Dela Coleta, da Universidade Federal de Uberlândia, e também da Prof ${ }^{a}$. Dra. Marília Dela Coleta.

Participaram também do processo de capacitação outros docentes de 5 Departamentos Clínicos da FMRP (Cirurgia, Clínica Médica, Ginecologia e Obstetrícia, Medicina Social e Pediatria e Puericultura) e que constituíram equipes departamentais, corresponsáveis pelo planejamento, elaboração, aplicação e correção das diferentes provas.

Durante os 8 anos em que a avaliação foi realizada, fizeram parte das equipes departamentais, uma ou mais vezes, os seguintes docentes ou colaboradores: Afonso Dinis Costa Passos, Aldaisa Cassanho Forster, Amábile Rodrigues X. Manço, Antonio Alberto Nogueira, Breno José G. Simões, Carlos Eli Piccinato, 
Eduardo Antonio Donadi, Eduardo Ferriolli, Elisabeth Meloni Vieira, Francisco Eulógio Martinez, Geraldo Duarte, Hélio Humberto A. Carrara, Heloísa Bettiol, Jarbas Leite Nogueira, José Fernando de C. Figueiredo, José Ivan de Andrade, José Sebastião dos Santos, Juan Stuardo Y. Rocha, Júlio C. Morigutti, Lívia Carvalho Galvão, Luiz Donizetti Stracieri, Marcio Dantas, Marcos Dias de Moura, Maria Célia Cervi, Marisa Marcia Mussi Pinhata, Omar Feres, Rosana Maria dos Reis, Salim Moysés Jorge, Sandro Scarpellini, Silvana Maria Quintana, Sílvio Tucci Júnior, Uilho Antonio Gomes e Yvone Avaloni de M. V. A. Vicente.

O objetivo primordial do processo de avaliação foi o de permitir uma análise comparativa do desempenho dos formandos da FMRP-USP em habilidades e competências, abrangendo as esferas cognitiva, psicomotora, clínica e afetiva, nas duas estruturas curriculares (antiga e nova). A principal tarefa do Grupo de Trabalho para Avaliação terminou ao se avaliarem as 3 últimas turmas da estrutura curricular antiga e as 3 primeiras turmas do currículo novo.

No seu conjunto, os resultados do processo de avaliação terminal dos graduandos da FMRP-USP (apresentados sob a forma de relatório entregue à Comissão de Graduação e apresentado em sessão da Congregação) indicaram que a implantação da atual estrutura curricular associou-se a aumento da aquisição de conhecimentos nas áreas mais gerais da atuação médica e melhora significativa do desempenho prático em habilidades clínicas fundamentais e, sobretudo, nas habilidades psicomotoras, envolvidas na realização de procedimentos. O novo currículo parece ter afetado de modo negativo a aquisição de habilidades cognitivas especiais e mais complexas, cujo domínio já era insuficiente na estrutura anterior. Não houve, também, modificação expressiva do grau de domínio dos objetivos educacionais da instituição, muito embora as diferenças encontradas foram, em sua maioria, favoráveis à nova estrutura curricular.

Outro aspecto estudado pelo grupo foi o das atitudes dos graduandos frente a aspectos relevantes da prática médica, e o que se verificou foi a ausência de efeito da restruturação curricular sobre as atitudes claramente positivas frente aos fatores psicológicos e sociais, envolvidos nas doenças orgânicas e às práticas de atenção primária. Do mesmo modo, não se verificou influência significativa das mudanças curriculares sobre atitudes pré-existentes de natureza conflitiva, em relação à doença mental e à morte.

O processo motivou o grupo a estudar outros aspectos relacionados com a formação médica, que poderiam estar interferindo nos resultados da avaliação. A análise dos dados, referentes a múltiplas vertentes abordadas, propiciou a elaboração de 32 comunicações (28 no Exterior e 4 no Brasil) em eventos científicos, relacionados ao ensino médico (Otawa Conference, 1994 e 1998; Association for Medical Education in Europe - AMEE - Meeting, 1995, 1996, 1997, 1998, 1999, 2000, 2001, 2002; Congresso Brasileiro de Educação Médica, 1995; Congresso Paulista de Educação Médica, 2000, 2002). Vinte e sete das comunicações tiveram seus resumos publicados e uma delas originou a publicação de um trabalho completo em anais de Congresso no Exterior. A produção bibliográfica do grupo incluiu, também, a publicação de 1 capítulo de livro (no Exterior) e 4 trabalhos completos ( 1 no Exterior), além 2 relatórios técnicos. Foram ainda encaminhados para publicação outros 3 trabalhos completos (1 no Exterior); um desses artigos já foi aceito pela Revista Brasileira de Educação Médica.

Ainda como resultado do envolvimento do grupo com o processo de avaliação, alguns de seus membros têm sido convidados para ministrar palestras em eventos nacionais (Congresso Paulista de Ensino Médico, CEDEM-USP) e no Exterior (Ibero-American Group da AMEE).

As atividades do grupo motivaram, também, o interesse de outras instituições nacionais de ensino superior, que enviaram observadores para acompanhar os trabalhos de avaliação (FMUSP, FAMEMA, FAMERP, PUCCAMP), ou que convidaram membros do grupo para palestras e apresentações parciais dos resultados (UNIFESP, FMUFRGS, UNICAMP, FFCMRGS e PUCCAMP).

Deve ser enfatizado o grande apoio que a instituição forneceu para a capacitação dos membros do grupo de trabalho e docentes que colaboraram, financiando e facilitando a participação em workshops na área de ensino médico (5 no exterior e 2 no Brasil) e estimulando a realização de visitas científicas a outras instituições de ensino, especialmente as que introduziram inovações nos processos de ensino/aprendizagem e avaliação (University of Toronto e McMaster University, Estados Unidos, Canadá, Chulalongkorn University, na Tailândia, Southern Illinois School of Medicine, University of Massachussets, Harvard Medical School, Universitat de Barcelona, Espanha, Université de Sherbrooke, Universidade de Lisboa, Portugal, University of Limburg, em Maastrich, Holanda, Universitá degli studi di Verona, Itália). 
Esses investimentos institucionais propiciaram a criação de 2 disciplinas no Curso de Pós-Graduação, relacionadas à Educação Médica (RCM-5799 - Avaliação de Competências Clínicas e ROT- 5721- Métodos de Ensino em Pequenos Grupos) e a reformulação de uma outra (ROT-5719 - Aspectos Didáticos e Pedagógicos em Especialidades Clínico-cirúrgicas).

Ainda como conseqüência da capacitação desse grupo em aspectos gerais, relacionados com a Educação Médica, atualmente, por designação do Sr. Diretor da FMRP, seus componentes docentes, junta- mente com os Profs. Drs. Afonso Dinis Costa Passos e Sérgio Britto Garcia, foram incorporados ao Grupo de Assessores do CAEP, centro, atualmente, coordenado pelo Prof. Dr. Ricardo Brandt de Oliveira.

Todo o grupo, assim como a equipe técnica do CAEP (atualmente composta pela Psicóloga Maria de Fátima Aveiro Colares e pela Pedagoga Cristiane Martins Peres), desenvolvem, no momento, projeto para implantação de Sistema de Tutorias na Faculdade de Medicina de Ribeirão Preto da Universidade de São Paulo. 